\title{
Concurrence of Sweet's Syndrome, Pathergy Phenomenon and Erythema Nodosum-like Lesions
}

Jianjun Qiao ${ }^{1}$

Yinhua $\mathrm{Wu}^{1}$

\author{
Yan Wang ${ }^{1}$ \\ Hong Fang ${ }^{1}$
}

Juan $\mathrm{Bai}^{1}$

\begin{abstract}
We report the case of a 54-year-old woman with concurrent Sweet's syndrome, pathergy phenomenon and eythema nodosum-like lesions associated with suppurative tonsillitis. Tender, violaceous and highly edematous papules and plaques were detected on the forearms and legs, some of which were pseudovesicular. Similar edematous papules were detected on the dorsum of the hands at the sites of intravenous injection. Biopsies of a plaque on the forearm and a papule at the site of intravenous injection confirmed the presence of Sweet's syndrome. Multiple, tender, violet-red, subcutaneous erythematous nodules were revealed on the legs. A biopsy taken from a nodule on the leg revealed septal panniculitis. To our knowledge, no patient with concurrent Sweet's syndrome, pathergy phenomenon, and erythema nodosum-lesions had been reported yet in previous literature.
\end{abstract}

Keywords: Concurrent symptoms; Erythema nodosum; Sweet syndrome

\section{INTRODUCTION}

Sweet's syndrome, also named as acute febrile neutrophilic dermatosis, is clinically characterized by the abrupt development of painful erythematous papules and plaques that can be pseudovesicular. Fever, arthralgias, leukocytosis with neutrophilia, elevated Creactive protein and erythrocyte sedimentation rate may occur. ${ }^{1-4}$ Erythema nodosum is a form of septal granulomatous panniculitis characterized by abruptly developing painful red nodules mainly over the pretibial area. ${ }^{1,5}$ Concurrence of the two reactive cutaneous conditions seems to be infrequently reported.

Pathergy is defined as an induction of dermatosis-associated skin lesions appearing at sites of skin trauma. ${ }^{2,6}$ The pathergy phenomenon in Sweet's syndrome patients is rarely reported.

We reported the case of a patient with simultaneous occurrence of Sweet's syndrome associated with pathergy and erythema nodosum-like lesions. To our knowledge, no patient with concurrent Sweet's syndrome, pathergy phenomenon, and erythema nodosum-lesions had yet been reported in previous literature.

\section{CASE REPORT}

A 54-year-old woman presented with a 7-day history of painful erythematous lesions on the lower and upper extremities. These lesions were preceded by a one-day history of sore throat and fever. Suppurative tonsillitis had been confirmed by an otolaryngologist. Her past medical history did not reveal any previous disease. Intravenous levofloxacin had been given to control the tonsillitis. Lesions similar to those on the upper extremities occurred on the dorsum of the hands at the sites of intravenous injection.

Physical examination revealed a temperature of $39^{\circ} \mathrm{C}$. Tender, violaceous and highly edematous papules and plaques were detected on the forearms and legs, some of which were pseudovesicular (Figures 1A and 1B). Similar edematous papules were detected on the dorsum of the hands at the sites of intravenous injection (Figure 1C). Multiple, tender, violet-red, subcutaneous erythematous nodules were revealed on the legs (Figure 1D).

Her peripheral blood neutrophil count was elevated at $11.1 \times 10^{9} / \mathrm{L}$ [normal, $(4$ to 10$) \times 10^{9} / \mathrm{L}$, with a neutrophilic predominance of $81.4 \%$. She had an elevated C-reactive protein level ( $27 \mathrm{mg} / \mathrm{L}$; normal, 0 to $8 \mathrm{mg} / \mathrm{L}$ ) and erythro-

Received on 05.10.2013.

Approved by the Advisory Board and accepted for publication on 20.11.2013.

Work performed at the Department of Dermatology, The First Affiliated Hospital, College of Medicine, Zhejiang University - Zhejiang Province , China.

Conflict of interest: None

Financial funding: None

The First Affiliated Hospital, College of Medicine, Zhejiang University - Zhejiang Province, China. 

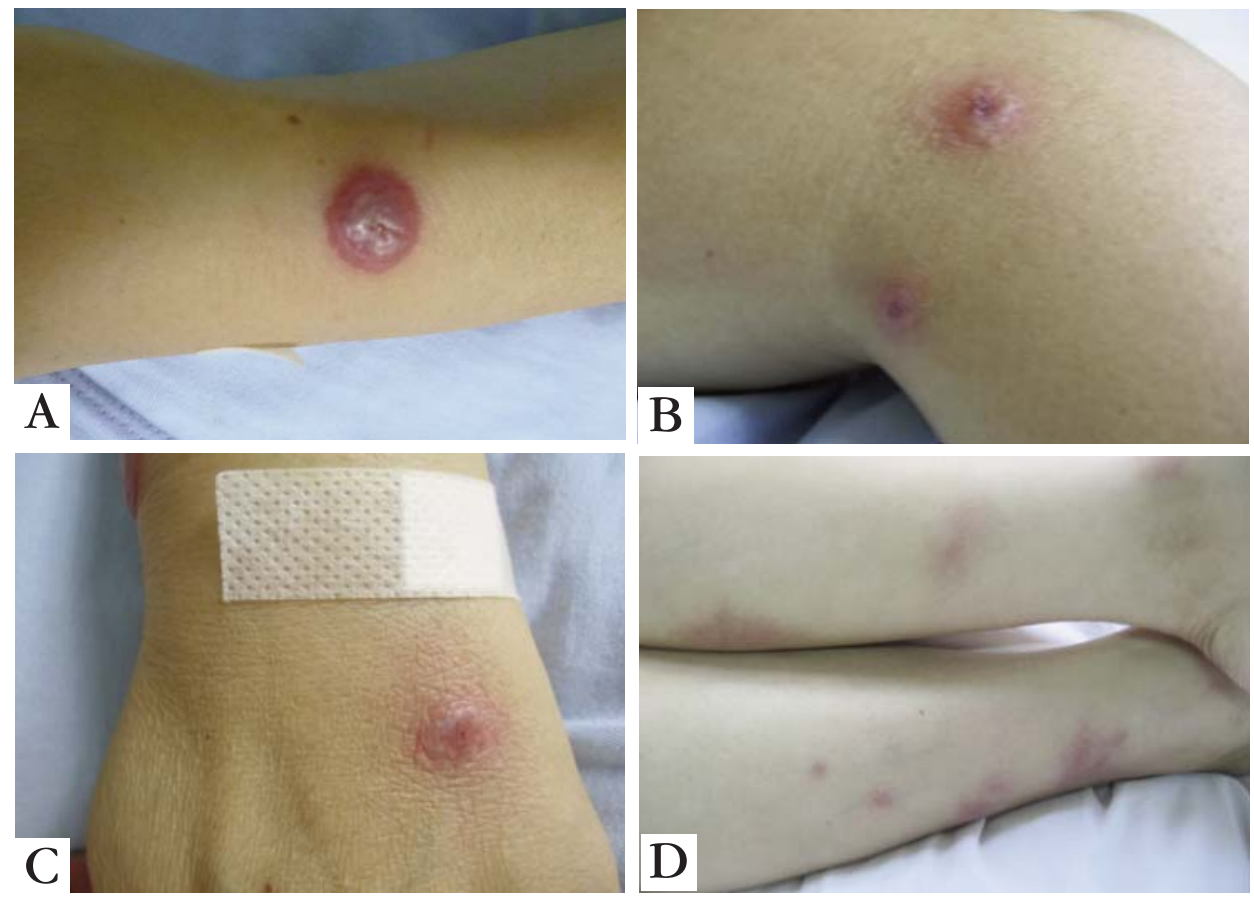

Figure 1:

A. Eruptions of Sweet's syndrome with pathergy phenomenon and erythema nodosumlike lesions. (a) Pseudovesicular plaques on the arm and the leg; $\mathbf{B}$. Pseudovesicular plaques on the leg; C. Pathergy phenomenon at the site of intravenous injection on the dorsum of the left hand; D. Erythema n o d o s u m - like lesions on the legs
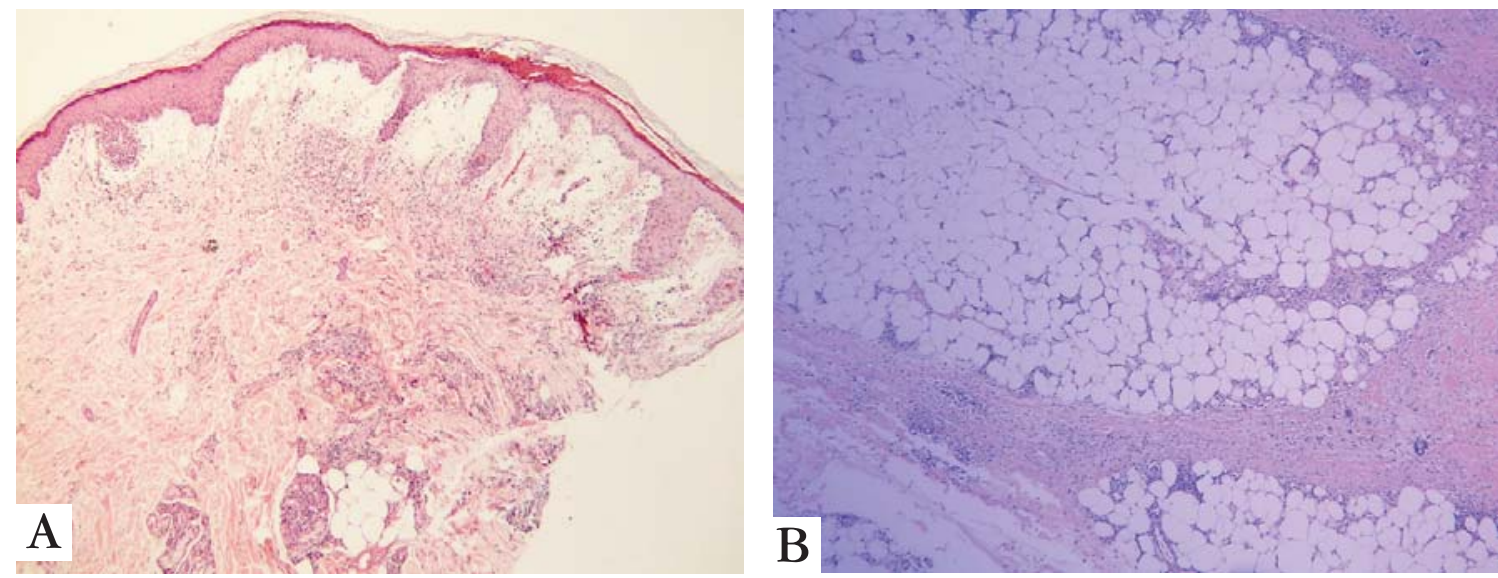

FIgURE 2: A. Histopathological manifestation of Sweet's syndrome. Prominent edema in the upper dermis and dense neutrophilic infiltrate (H\&E, original magnification x40); B. Biopsy of erythema nodosum-like lesion showed septal panniculitis with infiltrate of neutrophils, histiocytes and giant cells (H\&E, original magnification x100)

cyte sedimentation rate $(31 \mathrm{~mm} / \mathrm{h}$; normal, 0 to $20 \mathrm{~mm} / \mathrm{h})$.

Skin a plaque on the forearm and a papule at the site of intravenous injection, were consistent with Sweet's syndrome. They showed prominent edema on the upper dermis and a diffuse neutrophil-rich infiltrate in the upper and mid dermis with leukocytoclasis (Figure 2A). A biopsy taken from a nodule on the leg revealed septal panniculitis with infiltrate of neutrophils, histiocytes and giant cells. The pathological change was consistent with erythema nodosum (Figure $2 \mathrm{~B}$ ).

With these clinical and histopathologic findings, a diagnosis of Sweet's syndrome with pathergy phenomenon and erythema nodosum-like lesions was made. 
The patient was given methylprednisolone at a dose of $40 \mathrm{mg}$ per day to control the cutaneous conditions. She had a dramatic response with complete resolution of her fever and skin lesions in 10 days. The peripheral blood leukocyte count, C-reactive protein level, and erythrocyte sedimentation rate recovered normal range.

\section{DISCUSSION}

Our patient presented with two unusual clinical features. First, Sweet's syndrome and erythema nodosum-like lesions appeared simultaneously. Second, the obvious cutaneous pathergy occurred, i.e. lesions occurred at the sites of intravenous injection. To our knowledge, no patient of Sweet's syndrome with pathergy phenomenon and concurrent erythema nodosum-lesions had yet been reported in previous literature.

Sweet's syndrome and erythema nodosum share many similar clinical and histological features. ${ }^{1-3,7,8}$ Both have acute onset and occur in association with many of the same systemic conditions, such as upper respiratory infections, medications, hematologic malignancies, and autoimmune disease. ${ }^{2,5,8}$ The two conditions are responsive to similar therapeutic drugs as systemic glucocortioids or potassium iodide. ${ }^{25,7,9}$ Recently 16 cases of biopsy-confirmed occurrence of Sweet's syndrome and erythema nodosum-like lesions were well reviewed.?

\section{REFERENCES}

1. Bonamigo RR, Razera F, Olm GS. Neutrophilic dermatoses: part I. An Bras Dermatol. 2011;86:11-25.

2. Cohen PR. Sweet's syndrome--a comprehensive review of an acute febrile neutrophilic dermatosis. Orphanet J Rare Dis. 2007;2:34.

3. Eleuterio IA, Tiussi RM, Delmaestro D, Diniz LM, Lucas EA. Sweet's syndrome: clinicopathological features of patients treated from 1997 to 2009 at Cassiano Antonio Moraes University Hospital - Vitoria (Espirito Santo). An Bras Dermatol. 2012;87:450-5.

4. Rochael MC, Pantaleão L, Vilar EA, Zacaron LH, Spada EQ, Xavier MH, et al. Sweet's syndrome: study of 73 cases, emphasizing histopathological findings. An Bras Dermatol. 2011;86:702-7.

5. Mana J, Marcoval J. Erythema nodosum. Clin Dermatol. 2007;25:288-94.

6. Varol A, Seifert 0 , Anderson CD. The skin pathergy test: innately useful? Arch Dermatol Res. 2010;302:155-68.

7. Tabanlioğlu D, Boztepe G, Erkin G, Gököz 0, Karaduman A. Sweet's syndrome and erythema nodosum: a companionship or a spectrum?--a case report with review of the literature. Int J Dermatol. 2010;49:62-6.

8. Ginarte M, Toribio J. Sweet's syndrome and erythema nodosum: two neutrophilic dermatoses? Clin Rheumatol. 2007;26:1215-6.

9. Wasson S, Govindarajan G, Folzenlogen D. Concurrent occurrence of Sweet's syndrome and erythema nodosum: an overlap in the spectrum of reactive dermatoses. Clin Rheumatol. 2006;25:268-72.

10. Guhl G, García-Díez A. Subcutaneous sweet syndrome. Dermatol Clin. 2008;26:541-51, viii-ix.
Nonetheless there are some differences between Sweet's syndrome and erythema nodosum. ${ }^{1,2,5,7,9}$ Sweet's syndrome lesions mainly involve the upper part of the body - head, neck, shoulder and trunk, while erythema nodosum lesions are characteristically seen on the pretibial region., ${ }^{2,5}$ Erythema nodosum lesions reveal deeper inflammatory cell infiltrate in subcutaneous adipose tissue, though this infiltrate is more superficial in dermis in Sweet's syndrome lesions. ${ }^{2,5}$

A recently proposed entity subcutaneous Sweet's syndrome referred to those whose neutrophilic infiltrate is located in the subcutaneous adipose tissue only, including the lobule, septa, or both. 10 According to this proposal, erythema nodosumlike lesions in Sweet's syndrome patients may be a subcutaneous variant of Sweet's syndrome.

This case also illustrates an association of the pathergy phenomenon with Sweet's syndrome. Pathergy in Sweet's syndrome patients is rarely reported. ${ }^{2}$ The pathogenesis of the pathergy in Sweet's syndrome is not determined. Sweet's syndrome may occur at the sites of biopsy, vaccination, intravenous catheter placement, injection, as well as insect bites and cat scratches. ${ }^{2}$ Sweet's syndrome lesions can also occur in areas that have received radiation therapy, locations that have been in contact with sensitizing antigens, places that have received pressure, and regions damaged by sunburn. ${ }^{2}$ In our case, the lesions were clearly related to intravenous injection.

How to cite this article: Qiao J, Wang Y, Bai J, Wu Y, Fang H. Concurrence of Sweet's Syndrome, Pathergy Phenomenon and Erythema Nodosum-like Lesions. An Bras Dermatol. 2015;90(2):237-9. 